\title{
Application of the Remote Sensing Technology in the Line Arrangement of Shallow Artificial Seismic Exploration
}

\author{
Weidong Sun \\ School of the Earth Sciences and Resources, China University of Geosciences, Beijing 100083, China \\ Center of Information, Xinjiang Geology and Mining Bureau, Urumqi 830000, China \\ Tel: 86-991-485-6308 E-mail: sunwindabc@tom.com \\ Wei Zhang (Corresponding Auhtor) \\ China Aero Geophysical Survey \& Remote Sensing Center for Land and Resources \\ Beijing 100083, China
}

Tel: 86-10-8232-9026Ｅ-mail: zhangwei@agrs.cn

\begin{abstract}
The remote sensing image can not only show the panorama information of the fault distribution, but also offer the information about the geology, physiognomy and digital elevation with high precision. In this paper we took the Hangzhou area as the example and systematically introduced the application of the remote sensing technology in the optimal design of the shallow artificial seismic line, and it was very important to optimize the seismic line distribution of the shallow artificial seismic exploitation, perfect the quality of the seismic materials, enhance the work efficiency and reduce the exploitation costs.
\end{abstract}

Keywords: Remote sensing image, Seismic line, Optimization design, Hangzhou

The remote sensing doesn't contact the objects, and it utilizes the visible light detector, infrared detector or the microwave detector to detect the electromagnetic wave characteristics of the objects on the earth's surface through many technologies such as photograph scanning, information induction, information transportation and information processing from the remote platform, the upper air even the outer space, and find out the natural status, the characteristics and evolvement of the object. The remote sensing image can not only subjectively, really and comprehensively offer the physiognomic characteristics, the lithology of the earth's surface, the aquosity, the loose, the attitude of stratum, the human traffic, digital elevation and 3D real-time simulation, but also reflect the information characteristics of the concealed geological structure under certain depth (Zhang, 2007, P.10-14). Combined with GPS, GIS, DEM and other technologies, the image information can offer large conveniences for the seismic prospecting and largely enhance the timeliness of the seismic exploitation.

The plain area of Hangzhou City occupies $70 \%$ of the whole city area, and most areas are covered by the Quaternary unconsolidated sediments. The region is the multiple-grown concealed fault, and according to past field seismic geological survey and the shallow artificial seismic exploration data, the active fault in the Late Pleistocene epoch through the whole region may exist in the Hangzhou area. In A.D.929, destructive earthquake of 5 magnitude happened in Hangzhou. Therefore, Hangzhou municipal government developed the work of "Hangzhou Municipal Earthquake Active fault Exploitation and Earthquake Fatalness Evaluation" in 2005 to detect whether there was the concealed active fault under Hangzhou City and evaluate the fatalness of the earthquake, and accordingly adopted the feasible measures protecting against and eliminating earthquake disasters, and offered the references for the scientific decisions of the city planning and social development. The base of the "active fault" exploitation is to detect the position of Hangzhou main fault concealed sect, the layer position and the depth of the fault, and analyze the possible activity of the fault. The application of the remote sensing image information in the line arrangement of shallow artificial seismic exploration is mainly embodied in the following aspects.

\section{Optimization of seismic lines}

The cost of shallow artificial seismic exploration is higher, and each kilometer construction plant needs 30,000 Yuan, and the price will be higher when the construction plant is in the city zone, because it needs large numbers of finance, manpower and material resources. To enhance the efficiency of the seismic prospecting and reduce the exploitation costs, the seismic exploitation must be deployed in the regions with the exploitation necessity and construction conditions and the distribution principle of "avoiding the highness and dwelling on the lowness, avoiding the gravel and 
dwelling on the rock, avoiding the mixed and dwelling on the simply, avoiding the dry and dwelling on the wet, avoiding the steep and dwelling on the slight (Hu, 2005, P.316-317)". The remote sensing image can not only show the spacial distribution of the active fault and intuitively open out the image information of the renewed faulting, but also offering abundant information to analyze the active status of the fault and its relation with the earthquake and identify the sect of the earthquake gestation. In the concrete distribution of the seismic line, we can optimize the seismic lines for the conformation characteristics, landform characteristics, the earth's surface type and the vegetation, live up to avoid the construction difficulties brought by the highly complex landform, and complete the deployment of the seismic lines, and better control and detect the object faulting.

\section{Selection of construction plants}

The floor geological survey in the seismic exploitation area is the important base for the seismic engineering design, signal acquirement and data processing. It is very difficult to implement the seismic exploitation in the region of Hangzhou. First, the depth of the Quaternary covering layer in the Hangzhou area is very thin $(0 \sim 100 \mathrm{~m})$, and in the local lots, the stone heads are bared or half bared, and they belong to the super shallow seismic exploitation, and the technical difficulty of the seismic prospecting is higher. Second, most seismic lines are in the city zone and the outskirts of Hangzhou, and in those places, the buildings are dense, the roads extend in all directions, which could not only bring inconveniences for the field construction, but the jamming is serious. Third, the short seismic section length is only about 2 kilometers, and because of the plane swing of the fault, some short sections will not span the objective fault. In addition, the Qiantangjiang River and the Great Canal traverse the Hangzhou City, which will bring certain influences for the seismic construction quality. Therefore, certain warps will exist between the seismic lines of the design in the room and the construction lines, and we need to develop the practical field geological survey before the construction. The work of field survey is large, and the labor intension is high, the work efficiency is low, the work time is long and the capital devotion is large, the comprehensive concept is lacked and the pertinence is bad and control precision is low. The high precision remote sensing image can comprehensively offer the relative distribution information about the building, the road, the vegetation and the water system, and the overlay analysis of the remote sensing image and the DEM can simulate the 3D dynamic flying exploration in the room, quickly select the construction plant and the optimal plant, avoid the disjoint of the room seismic exploitation distribution and the field seismic exploitation distribution, and avoid the bad plan of the field seismic lines distribution, and avoid influencing the selection of the shot-point and the demodulator probe and the material quality (Zhao, 2005, P.33-35).

\section{Optimization of seismic observation system}

The main difficulty of the seismic data field acquisition in Hangzhou is that the seismic geological condition is very complex. To obtain better original single-shot record and perfect the quality of the seismic data, we should select multiple experiments points to implement the comparison experiment about inspiring condition and accepting condition in the construction region before the construction, and ensure that the shot-points are distributed in the region with better inspiring condition.

The remote sensing image contains abundant information such as the landform elevation, the stratum attitude, the lithology of earth's surface, the physiognomy and the vegetation, and relative humidity, and by these intuitive pictures and graph information, we can realize the pre-distribution of the seismic inspiring point, the seismic accepting point, and according to the change of the inspiring point and accepting point on the earth's surface, we can timely adjust the distribution density and interval, and make the inspiring point and the accepting point distribute on the earth's surface with single lithology, low-lying and flat landform, and relatively hard surface layer, which could optimize the inspiring parameters and enhance the SNR of data. Otherwise, the exact geological coordinate data and the elevation data offered by the remote sensing data can largely offer convenient for the field construction measurement and enhance the work efficiency.

\section{Optimization of seismic construction organization and logistic guarantee}

The information such as areal geology, physiognomy, humanity and traffic offered by the remote sensing image can not only be applied in the seismic acquisition technological design, but also offer the information service for the seismic construction organization, the implementation equipment selection, the work risk evaluation and reasonable encampment selection, transportation lines and logistical mode. Especially under the supports of GPS, GIS and DEM, the exact 3D dynamic image can make the seismic work organization, material supply and living supply more scientific and reasonable, which will exert effective function to enhance the seismic work efficiency, reduce the inefficient devotion and control the increase of cost.

Except for that, according to the image information offered by the remote sensing image with high precision, we can exactly quantitatively confirm the surface type and the range in the seismic construction region, and the type and the quantity of the seismic equipment needed, and objectively evaluate the engineering budge of the seismic acquisition. 


\section{Experiment results}

In the study, we took Hangzhou as the example, utilize the ETM+ satellite multiple-spectrum image, take the faults in Hangzhou as the main research objects, give prominence to the image characteristic of city fault through effective remote sensing image interpretation based on the image processing including atmospheric correction, geometric precision correction, de-noising and image enhancement, primarily confirm the position and distribution characteristics of the main faults in the region combined with the computer, and obtain the fault geology and physiognomy information in the width of 2 kilometers along the concealed fault, and the fault positioning precision is about $200 \mathrm{~m}$, which can offer the reference (Figure1) for distributing the line arrangement of shallow artificial seismic exploration, reduce the exploitation range, increase the exploitation points in the possible faults and enhance the exploitation precision. In the actual production, this study obtained better effect, largely enhance the work efficiency and correspondingly reduce the work cost.

\section{Conclusions}

Through utilizing the remote sensing image with high precision, combining with digital landform figure and DEM, adopting the exact geological information, digital elevation information, physiognomy information and surface lithology offered by the advanced remote sensing technology, and breaking through the view limitation of the field measurement, we can enhance the reasonability and the science character of the shoot-point distribution, perfect the quality of the seismic acquisition data, enhance the construction efficiency and reduce the exploitation costs.

\section{References}

$\mathrm{Hu}$, Yan, Zhang, Youyan, Ye, Yong, et al. (2005). Application of Remote Sensing to Optimizing of Seismic Line Arrangement. Xinjiang Petroleum Geology. No. 26 (3). P.316-317.

Zhang, Jingfa, Tao, Xiaxin, Tian, Yunfeng, et al. (2004). Application of remote sensing technique to exploration of active fault around urb. Journal of Natural Disasters. No. 13 (1). P.13-22.

Zhang, Wei, Chen, Hanlin, Li, Qimin, et al. (2007). Application of high resolution remote sensing technique to exploration of active fault of Hangzhou and its surroundings. Coal-geology \& Exploration. No. 35 (3). P.10-14.

Zhao, Bangliu, Du, Xiaodi, Zhang, Youyan, et al. (2005). Application of high resolution remote sensing images to the seismic exploration in complex surface area. Exploration Techniques. No. 2. P.33-35.

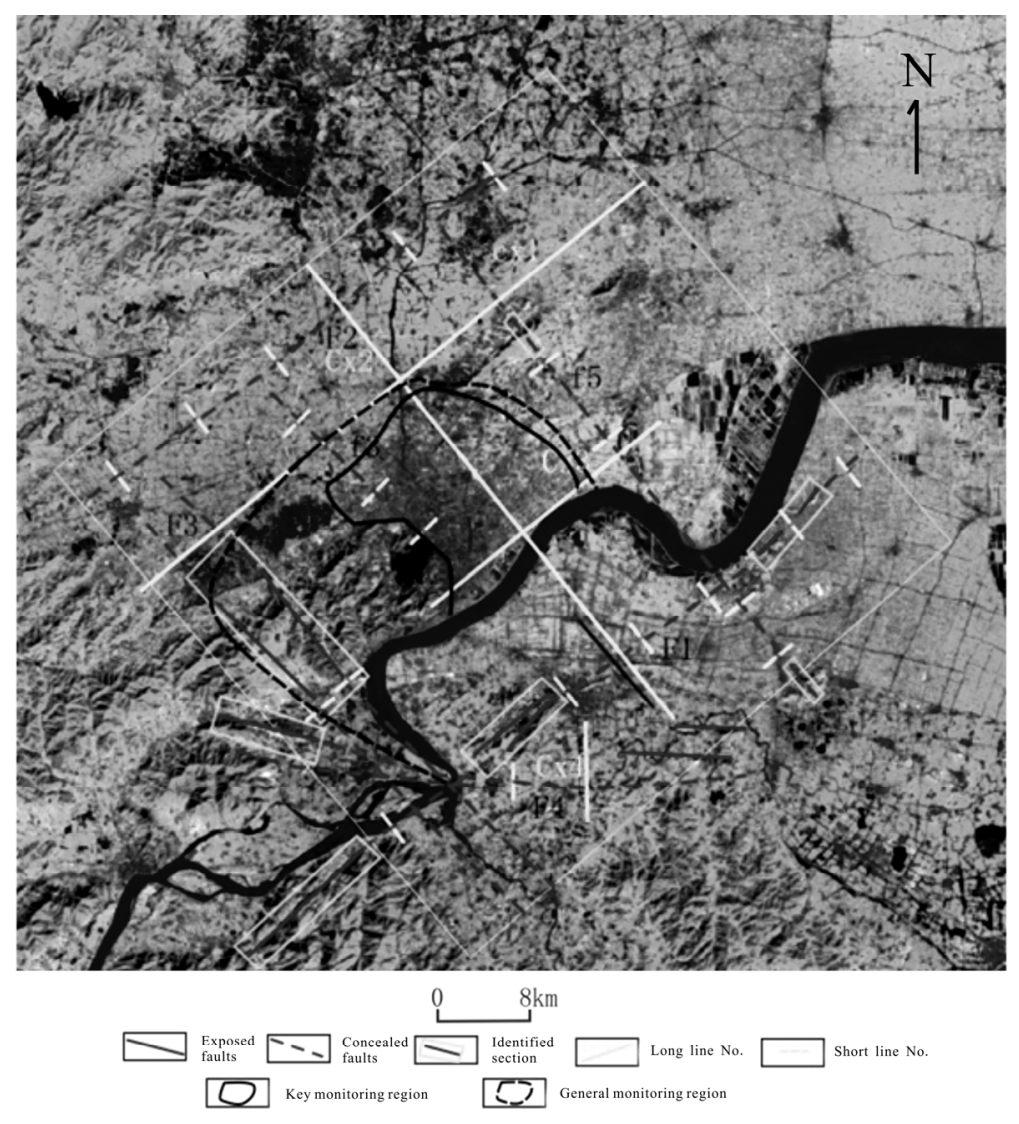

Figure 1. The Line Arrangement of Shallow Artificial Seismic Exploration in the Hangzhou Area 